\title{
Polymorphisms in CYP2C9 are associated with response to indomethacin among neonates with patent ductus arteriosus
}

\author{
Caitlin J. Smith ${ }^{1}$, Kelli K. Ryckman ${ }^{1}$, Timothy M. Bahr ${ }^{2,3}$ and John M. Dagle ${ }^{2}$
}

BACKGROUND: Patent ductus arteriosus (PDA) is a common complication seen in preterm infants. Indomethacin is routinely used to treat PDA. Evidence suggests that the response of indomethacin is highly heritable. This study investigated the association between single-nucleotide polymorphisms (SNPS) in CYP2C9 and the closure of PDA in response to indomethacin.

METHODS: Six SNPs in CYP2C9 were analyzed for association with indomethacin response. A case-control analysis was performed among neonates who responded to indomethacin (responders) and among those who required surgical ligation (non-responders). Independent transmission disequilibrium tests were performed among parent-child trios of responders and non-responders.

RESULTS: The G allele of rs2153628 was associated with increased odds of response to indomethacin in the casecontrol analysis (odds ratios (OR): 1.918, 95\% confidence interval (Cl): 1.056, 3.483). Among indomethacin responders, the $G$ allele of rs2153628 and the T allele of rs1799853 were overtransmitted from the parents to their child (OR: 2.667, 95\% $\mathrm{Cl}: 1.374,5.177$ and OR: 2.375, 95\% Cl: 1.040, 5.425, respectively), consistent with the case-control analysis.

CONCLUSION: We identified an association between two SNPs in CYP2C9, rs2153628 and rs1799853, and indomethacin response for the treatment of PDA. These findings suggest that response to indomethacin in the closure of PDA may be influenced by polymorphisms associated with altered indomethacin metabolism.

$\mathbf{T}$ he ductus arteriosus is a vital fetal structure connecting the aorta and the pulmonary artery, shunting blood from the pulmonary circulation (1). Patent ductus arteriosus (PDA) is a neonatal complication in which the ductus arteriosus fails to close, resulting in continued postnatal shunting of systemic and pulmonary circulations. It commonly affects very preterm infants, although it can also affect term infants (2). Although many PDAs close over time without treatment, active treatment for PDA ranges from nonsteroidal anti-inflammatory drugs (NSAIDs), including ibuprofen and indomethacin, to surgical ligation (3). Only two-thirds of neonates experience closure of PDA in response to indomethacin (4). Importantly, post-treatment plasma concentrations and interpatient pharmacokinetic differences have been shown to affect closure rates. Pharmacokinetic studies demonstrate considerable variability in indomethacin clearing among preterm infants with PDA $(5,6)$. Furthermore, PDA requiring indomethacin therapy has been reported as highly heritable, as has failure to respond to indomethacin therapy (7).

The cytochrome P450 gene family encodes a variety of enzymes that are critical for drug metabolism. The importance of these enzymes in drug metabolism, and their altered efficiency by genetic variation, has been known for decades (8). Cytochrome P450 enzymes are largely responsible for the metabolism of NSAIDs. In particular, the enzyme encoded by the CYP2C9 gene is known to metabolize many NSAIDs (9). In vitro studies have demonstrated that CYP2C9 is the major catalyst for metabolism of indomethacin to a product that accounts for $40-55 \%$ of total drug elimination in the urine (10). CYP2C9 is highly polymorphic with many functional variants of pharmacological importance. Most of the described polymorphisms are associated with reduced enzyme activity and therefore with decreased drug metabolism (9). Although the effects of these variants on drugs with narrow therapeutic indexes, such as phenytoin and warfarin, make up a majority of the pharmacogenetic literature, the effect of variations in the gene on NSAID metabolism and therapy has also been described (11).

Given that response to indomethacin has been shown to be heritable, and polymorphisms in CYP2C9 are known to affect NSAID metabolism, we investigated the association between specific polymorphisms in CYP2C9 and its response to indomethacin, as defined by the closure of PDA without surgery.

\section{METHODS}

\section{Study Population}

This study population was chosen from an existing cohort of infants admitted to the Neonatal Intensive Care Unit at the University of Iowa Children's Hospital between 1999 and 2015. Infants in the

\footnotetext{
${ }^{1}$ Department of Epidemiology, University of lowa, lowa City, lowa; ${ }^{2}$ Division of Neonatology, Department of Pediatrics, University of lowa, lowa City, lowa. Correspondence: Caitlin J. Smith (caitlin-smith-1@uiowa.edu)

${ }^{3}$ Current affiliation: Phoenix Children's Hospital, Phoenix, Arizona

Received 3 October 2016; accepted 15 April 2017; advance online publication 5 July 2017. doi:10.1038/pr.2017.145
} 
initial cohort were recruited as part of an initiative to create a research biobank of biologic material (blood and/or buccal swabs) from infants for subsequent use in investigations into the genetic contributions to neonatal conditions. Parental informed consent was obtained for inclusion in this biobank. Current study participants were parent-child trios of preterm singleton neonates ( $<32$ weeks' gestation) with PDA. All neonates in these analyses received indomethacin for the treatment of PDA following diagnosis by standard echocardiography around 1 week of life. Response to indomethacin was determined based on requiring surgery, in which surgical ligation was considered a non-response to indomethacin and no surgical ligation was considered a response to indomethacin. All study protocols and procedures were approved by the University of Iowa Institutional Review Board.

\section{Marker Selection}

Six SNPs located in CYP2C9 were selected for genotyping using the Tagger function in Haploview (rs4918758, rs1799853, rs2253635, rs4086116, rs1505, and rs2153628) (12,13). SNPs were selected to provide $>85 \%$ gene coverage. Six SNPs were selected with a minimum minor allele frequency of 0.20 . These SNPs provided an estimated coverage of $86 \%$ of the alleles of 22 SNPs located within CYP2C9.

\section{Genotyping}

Subjects were genotyped for six SNPs using TaqMan assays (Applied Biosystems, Foster City, CA) using the EP1 SNP Genotyping System and GT 192.24 Dynamic Array Integrated Fluidic Circuits (Fluidigm, San Francisco, CA). All genotyping reactions were performed according to the standard protocol provided by Fluidigm. Three CEPH-CEU individuals (1000 Genomes Project) served as positive controls and double-distilled water served as a negative control.

\section{Statistical Analysis}

Statistical analyses were performed using Plink software (Broad Institute, Cambridge, MA) and SAS version 9.4 (Cary, NC). SNP markers were assessed for Hardy-Weinberg equilibrium and were excluded from subsequent analyses with $P<0.01$. Individual SNPs and subjects were examined for genotyping efficiency and were excluded from subsequent analyses for genotyping efficiency $<95 \%$.

SNPs were analyzed for association with response to indomethacin in several ways. First, a case-control analysis was performed, in which cases were defined as neonates who received indomethacin and did not require surgery (responders) and controls were defined as neonates who received indomethacin and required surgery (nonresponders). Second, a transmission disequilibrium test (TDT) (14) was run among parent-child trios of neonates who did not respond to indomethacin and required surgery ("Non-responders TDT"). Third, a TDT was run among parent-child trios of neonates who received indomethacin and did not require surgery ("Responders TDT"). Odds ratios (ORs) and 95\% confidence intervals (95\% CIs) are reported for allelic tests. Haplotypes were tested for association in all three analyses.

\section{RESULTS}

All SNPs met Hardy-Weinberg equilibrium. All SNPs met a genotyping efficiency of $>95 \%$. Nineteen subjects were excluded for having a genotyping efficiency of $<95 \%$. Demographic characteristics are presented in Table $\mathbf{1}$. Responders and non-responders differed by gestational age, birth weight, and bronchopulmonary dysplasia status. Responders and non-responders did not differ by race, ethnicity, blood culture-positive sepsis within the first 21 days of life, intraventricular hemorrhage, necrotizing enterocolitis, respiratory distress syndrome, or retinopathy of prematurity.

Table 1. Demographic characteristics of responders and non-responders

\begin{tabular}{lccc}
\hline Variable & $\begin{array}{c}\text { Responders } \\
(N=96)\end{array}$ & $\begin{array}{c}\text { Non-responders } \\
(N=52)\end{array}$ & $P$ value \\
\hline Gestational age (weeks) $)^{\mathrm{a}}$ & $26.9(2.29)$ & $25.3(1.75)$ & $<0.0001$ \\
${\text { Birth weight }(\mathrm{g})^{\mathrm{a}}}^{1,012.9}$ & $782.5(201.9)$ & $<0.0001$ \\
& $(355.8)$ & &
\end{tabular}

Gender

0.702

Female

$43(45 \%)$

25 (48\%)

Male

$53(55 \%)$

$27(52 \%)$

Race

\section{Black/AA}

White

Other

Ethnicity

Hispanic/Latino

Non-Hispanic/Latino

Sepsis

Blood culture-positive

No

$B P D$

$$
\text { Yes }
$$

No

IVH

\section{Grade I}

Grade II

Grade III

Grade IV

None

RDS

$$
\text { Yes }
$$

No

NEC

$$
\text { Yes }
$$

No

$R O P$

Stage I

Stage II

Stage III

No

African American BPD, bor

dysplasia; IVH, intraventricular hemorrhage; NEC, necrotizing enterocolitis; RDS, respiratory distress syndrome; ROP, retinopathy of prematurity.

aifferences between responders and non-responders were analyzed by $t$-test. All other comparisons were made using $x^{2}$-test. 
Articles | Smith et al.

Table 2. Results of SNP-outcome association analyses

\begin{tabular}{ccccc}
\hline Analysis type & SNP & Effect allele & OR $(95 \% \mathrm{Cl})^{\mathrm{a}}$ & $P$ \\
\hline Case-control ( $\mathrm{N}=96$ cases, 52 controls $)$ & & \\
rs4918758 & $\mathrm{C}$ & $0.843(0.510,1.395)$ & 0.507 \\
rs2253635 & $\mathrm{A}$ & $0.898(0.548,1.469)$ & 0.667 \\
rs1799853 & $\mathrm{T}$ & $1.727(0.780,3.827)$ & 0.174 \\
rs4086116 & $\mathrm{T}$ & $1.179(0.634,2.191)$ & 0.602 \\
rs2153628 & $\mathbf{G}$ & $\mathbf{1 . 9 1 8 ( 1 . 0 5 6 ,}$ & $\mathbf{0 . 0 3 1}$ \\
& & & $\mathbf{3 . 4 8 3})$ & \\
rs1505 & $\mathrm{C}$ & $0.823(0.497,1.363)$ & 0.449
\end{tabular}

Non-responders TDT ( $\mathrm{N}=46$ families)

$\begin{array}{llll}\text { rs4918758 } & C & 0.867(0.412,1.821) & 0.706 \\ \text { rs2253635 } & \text { G } & 1.083(0.494,2.374) & 0.842 \\ \text { rs1799853 } & \text { T } & 0.500(0.188,1.332) & 0.157 \\ \text { rs4086116 } & \text { T } & 0.692(0.296,1.62) & 0.394 \\ \text { rs2153628 } & \text { G } & 1.800(0.603,5.371) & 0.285 \\ \text { rs1505 } & \text { C } & 0.867(0.412,1.821) & 0.706\end{array}$

Responders TDT ( $\mathrm{N}=98$ families)

$\begin{array}{lccc}\text { rs4918758 } & \text { C } & 0.741(0.416,1.321) & 0.307 \\ \text { rs2253635 } & \text { G } & 0.750(0.442,1.273) & 0.285 \\ \text { rs1799853 } & \text { T } & \mathbf{2 . 3 7 5 ( 1 . 0 4 0 ,} & \mathbf{0 . 0 3 4} \\ & & \mathbf{5 . 4 2 5}) & \\ \text { rs4086116 } & \text { T } & 1.385(0.678,2.826) & 0.369 \\ \text { rs2153628 } & \text { G } & \mathbf{2 . 6 6 7 ( 1 . 3 7 4 ,} & \mathbf{0 . 0 0 3} \\ & & \mathbf{5 . 1 7 7 )} & \\ \text { rs1505 } & \text { C } & 0.778(0.440,1.376) & 0.387\end{array}$

$\mathrm{Cl}$, confidence interval; $\mathrm{OR}$, odds ratio; $\mathrm{P}, \mathrm{P}$ value; SNP, single-nucleotide poly-

morphism; TDT, transmission disequilibrium test.

${ }^{a}$ Allelic test.

Bold indicates $P<0.05$

Results of the statistical analyses are shown in Table 2. In all, 148 subjects were included in the case-control analysis (96 cases/responders and 52 controls/non-responders), where cases were defined as neonates who received indomethacin and did not require surgery (responders) and controls were defined as neonates who were treated with indomethacin and required surgery (non-responders). The G allele of rs2153628 was associated with increased odds of response to indomethacin (OR: 1.918, 95\% CI: 1.056, 3.483, $P=0.031$ ). The genotype frequencies of rs2153628 among responders were $47.9 \%, 46.9 \%$, and $5.2 \%$ for $A A, A G$, and $G G$, respectively. The genotype frequencies of rs2153628 among non-responders were $69.2 \%, 27.0 \%$, and $3.8 \%$ for $\mathrm{AA}, \mathrm{AG}$, and GG, respectively. Among the 96 responders, 53 were males and 43 were females. Among the 52 non-responders, 27 were males and 25 were females. Sex was not significantly associated with response to indomethacin (data not shown). Among the 96 responders, 79 were Non-Hispanic White, six were Non-Hispanic Black, and 11 were of another race/ ethnicity. Among the 52 non-responders, 41 were NonHispanic White, seven were Non-Hispanic Black, and four were of another race/ethnicity. The mean gestational age was 26.9 weeks among responders and 25.4 weeks among nonresponders. Gestational age was significantly higher among responders compared with that among non-responders $(P<0.0001$, data not shown). Adjusting for gestational age, the OR for rs2153628 remained similar at 1.834 (95\% CI: $0.951,0.3 .535)$, albeit not statistically significant $(P=0.070)$. The OR for rs1799853 was not significant after adjusting for GA $(P=0.272)$.

In all, 46 families (14 parent-child dyads, 32 parent-child trios, total $N=124$ ) were included in the "Non-responders TDT" analysis of neonates who did not respond to indomethacin, requiring surgery to ligate the ductus. No SNPs were significantly associated with requiring surgery $(P>0.05)$. Among the 46 neonates, 24 were males and 22 were females. Among the 46 neonates, 37 were Non-Hispanic White, six were Non-Hispanic Black, and three were of another race/ethnicity. The mean gestational age was 25.5 weeks.

In all, 98 families (39 parent-child dyads, 59 parent-child trios, total $N=255$ ) were included in the "Responders TDT" analysis of neonates who responded to indomethacin and thus did not require surgery. The $G$ allele of rs2153628, located in CYP2C9, was significantly overtransmitted from parents to a child who responded to indomethacin with the closure of PDA (OR: 2.667, 95\% CI: 1.374, 5.177, $P=0.003$ ). The genotype frequencies of rs2153628 among probands were $47.0 \%, 49.0 \%$, and $4.0 \%$ for $\mathrm{AA}, \mathrm{AG}$, and $\mathrm{GG}$, respectively. The genotype frequencies of rs 2153628 among parents were $62.7 \%, 33.5 \%$, and $3.7 \%$ for $\mathrm{AA}, \mathrm{AG}$, and $\mathrm{GG}$, respectively. The $\mathrm{T}$ allele of rs1799853, located in CYP2C9, was also significantly overtransmitted from parents to a child who responded to indomethacin with the closure of PDA (OR: 2.375, 95\% CI: $1.040,5.425, P=0.034)$. The genotype frequencies of rs1799853 among probands were $74.5 \%$, $23.5 \%$, and $2.0 \%$ for CC, CT, and $\mathrm{TT}$, respectively. The genotype frequencies of rs1799853 among parents were $77.0 \%, 22.4 \%$, and $0.6 \%$ for CC, CT, and TT, respectively. Among the 98 neonates, 57 were males and 41 were females. Among the 98 neonates, 82 were Non-Hispanic White, five were Non-Hispanic Black, and 11 were of another race/ethnicity. The mean gestational age was 26.9 weeks.

Haplotypes containing rs2153628 were associated with indomethacin response in the case-control analysis $(P<0.05)$, although they did not meet statistical significance by Bonferroni correction (data not shown). Haplotypes were not significantly associated with indomethacin response in the TDT analyses (data not shown).

\section{DISCUSSION}

In a case-control analysis and a family-based TDT of neonates who responded to indomethacin, the $G$ allele of rs2153628 was significantly associated with response to indomethacin $(P=0.031$ and $P=0.003$, respectively). The $\mathrm{T}$ 


\section{CYP2C9 associated with indomethacin Articles}

allele of rs1799853 was significantly associated with response to indomethacin in the TDT analysis $(P=0.034)$ and with the same direction of effect in the case-control analysis, albeit not statistically significant $(P=0.174)$. Although $\mathrm{rs} 2153628$ has not been described in association with NSAID metabolism, it has been identified as a predictor of warfarin dosage (15) and CYP2C9 is known to be an important enzyme in NSAID metabolism (9). Its presence in the borderline significant haplotypes of the case-control population further supports its effect on indomethacin response.

The CYP2C9 2 polymorphism, represented by rs 1799853 , encodes a change from arginine to cysteine and is associated with slower metabolism of warfarin, and is thus associated with a lower required dose (16). The same study demonstrated that rs1799853 is the main driving polymorphism determining warfarin dosage, although many polymorphisms in CYP2C9 are in perfect linkage disequilibrium with rs1799853 (ref. 16). The SNPs rs1799853 and rs2153628 are not linked, with an $R^{2}$ of 0.03 (ref. 12), suggesting that these loci confer independent effects.

This study is strengthened by its utilization of both population-based and family-based analytical methods, the results of which suggest consistent direction of effect of the findings. In addition, the PDA phenotypes are carefully curated to prevent the inclusion of PDA diagnosed in the first few days of life, before the spontaneous closure is still likely. Although rs1799853 and rs2153628 were not statistically significant in the TDT of infants undergoing surgery for PDA, which may be due to the small sample size, the result of no association is consistent with the case-control findings because the effect alleles are associated with odds of response to indomethacin, rather than odds of requiring surgery. We were underpowered to detect an OR less than 3.5 in the casecontrol analysis. Although this may have caused us to miss some significant effects, it would not affect our ability to identify the positive associations described with CYP2C9. Adjusting for gestational age reduced the significance of our findings. This could be because gestational age is a confounder of the relationship between CYP2C9 polymorphisms, or it could be due to the small sample size. A limitation of this study is the small sample size. Replication or metaanalysis in another population would help clarify the relationship between gestational age, CYP2C9 polymorphisms, and response to indomethacin.

Population stratification is unlikely to be present in this study population, as most subjects were non-Hispanic White. A limitation of our study is the retrospective design that does not allow measurements of indomethacin levels; thus, we cannot determine whether genetic variation in CYP2C9 actually alters the rate of indomethacin metabolism in association with the closure of PDA. Notably, CYP2C9 is also involved with the metabolism of ibuprofen; thus, the results of this study are likely generalizable to infants whose PDAs are treated with ibuprofen (9).

We demonstrate that two genetic polymorphisms are associated with response to indomethacin for the treatment of PDA in a case-control analysis of neonates who required surgery compared with neonates who responded to indomethacin and in a family-based TDT of neonates who responded to indomethacin. Given that these polymorphisms are located in a gene that encodes an enzyme for indomethacin metabolism, these findings identify candidate polymorphisms for a genetic screening tool for indomethacin treatment of PDA. These findings are supported by pharmacokinetic studies, which report high variability in indomethacin clearing among preterm infants with PDA. Shaffer et al. concluded that indomethacin clearance is solely due to individual pharmacokinetic differences regardless of weight (5). Smyth et al. also reported high interindividual variability, although indomethacin metabolism did vary by weight (6). Validation studies to demonstrate the value of these polymorphisms in predicting response to indomethacin should be performed and translated into clinical practice to reduce the morbidity associated with prolonged PDA and indomethacin toxicity in which indomethacin treatment is likely to be futile.

\section{STATEMENT OF FINANCIAL SUPPORT}

This research was also supported, in part, by the National Institutes of Health (HL109199, HD057192, HD052953, HD065786). The content is solely the responsibility of the authors and does not necessarily represent the official views of the National Institutes of Health.

Disclosure: The authors declare no conflict of interest.

\section{REFERENCES}

1. Dagle JM, Lepp NT, Cooper ME, et al. Determination of genetic predisposition to patent ductus arteriosus in preterm infants. Pediatrics 2009;123:1116-23.

2. Hajj H, Dagle JM. Genetics of patent ductus arteriosus susceptibility and treatment. Semin Perinatol 2012;36:98-104.

3. How Is Patent Ductus Arteriosus Treated? National Heart, Lung, and Blood Institute, 2011. Available at https://www.nhlbi.nih.gov/health/ health-topics/topics/pda/treatment.

4. Gersony WM, Peckham GJ, Ellison RC, Miettinen OS, Nadas AS. Effects of indomethacin in premature infants with patent ductus arteriosus: results of a national collaborative study. J Pediatr 1983;102:895-906.

5. Shaffer CL, Gal P, Ransom JL, et al. Effect of age and birth weight on indomethacin pharmacodynamics in neonates treated for patent ductus arteriosus. Crit Care Med 2002;30:343-8.

6. Smyth JM, Collier PS, Darwish M, et al. Intravenous indometacin in preterm infants with symptomatic patent ductus arteriosus. A population pharmacokinetic study. Br J Clin Pharmacol 2004;58:249-58.

7. Lavoie PM, Pham C, Jang KL. Heritability of bronchopulmonary dysplasia, defined according to the consensus statement of the national institutes of health. Pediatrics 2008;122:479-85.

8. Meyer UA, Zanger UM. Molecular mechanisms of genetic polymorphisms of drug metabolism. Annu Rev Pharmacol Toxicol 1997;37:269-96.

9. Van Booven D, Marsh S, McLeod H, et al. Cytochrome P450 2C9-CYP2C9. Pharmacogenet Genomics 2010;20:277-81.

10. Zhou SF, Zhou ZW, Yang LP, Cai JP. Substrates, inducers, inhibitors and structure-activity relationships of human Cytochrome P450 2C9 and implications in drug development. Curr Med Chem 2009;16: 3480-675.

11. Ali ZK, Kim RJ, Ysla FM. CYP2C9 polymorphisms: considerations in NSAID therapy. Curr Opin Drug Discov Dev 2009;12:108-4.

12. Barrett JC, Fry B, Maller J, Daly MJ. Haploview: analysis and visualization of LD and haplotype maps. Bioinformatics 2005;21:263-5. 


\section{Articles | Smith et al.}

13. de Bakker PI, Yelensky R, Pe'er I, Gabriel SB, Daly MJ, Altshuler D. Efficiency and power in genetic association studies. Nat Genet 2005;37: $1217-23$.

14. Spielman RS, McGinnis RE, Ewens WJ. Transmission test for linkage disequilibrium: the insulin gene region and insulin-dependent diabetes mellitus (IDDM). Am J Hum Genet 1993;52:506-16.
15. Wadelius M, Chen LY, Eriksson N, et al. Association of warfarin dose with genes involved in its action and metabolism. Hum Genet 2007;121: 23-34.

16. King BP, Khan TI, Aithal GP, Kamali F, Daly AK. Upstream and coding region CYP2C9 polymorphisms: correlation with warfarin dose and metabolism. Pharmacogenetics 2004;14:813-22. 